\title{
Повышение надежности и выхода годных: традиционные и новые подходы
}

\author{
М. Макушин ${ }^{1}$, В. Мартынов, д. т.н. ${ }^{2}$
}

УДК 621.382 | ВАК 05.27 .06

По мере масштабирования микроэлектронных технологий, освоения производства новых поколений приборов задача повышения надежности ИС и выхода годных становится все более актуальной. На фоне роста издержек проектирования, разработки технологического процесса и освоения в производстве перспективной продукции жесткие требования предъявляются к сокращению цикла от начала разработки до запуска изделий в серию. Вопрос не только в достижении максимально возможной рентабельности, но и в получении экономических выгод от раннего выхода на рынок (премиальная цена*). Для увеличения выхода годных производители постоянно совершенствуют существующие методы метрологии и тестирования, а такке создают новые.

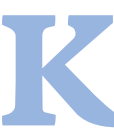

наиболее эффективным методикам метрологии и тестирования можно отнести способы проектирования под эксперимент (design of experiment, DoE), совместной оптимизации технологии и проектирования (desidn-technology со-optimization, DTCO), самовыравнивания в процессе многократного формирования рисунка с самосовмещением (self-aligned multi-patterning, SAMP) и т.д.

Относительно новая методика DoE представляет собой вид проектирования, направленного на описание и объяснение изменения информации в условиях, которые гипотетически отражают такое изменение. Методы DTCO и SAMP хорошо известны и широко используются.

Один из новейших подходов к увеличению выхода годных - использование технологий больших данных. По мере роста числа транзисторов на кристалле, этапов проектирования, отладки, верификации, контроля технологического процесса возникает все больший объем данных, которые благодаря оптимальному их использованию помогут совершенствовать производственный процесс. Общепринятого подхода еще не выработано, но производители признают данное направление перспективным и готовы тратить на развитие подобных методик значительные средства.

ЦНИИ «Электроника», главный специалист, mmackushin@gmail.com ФГБНУ "Аналитический Центр", проф., Минобрнауки РФ, эксперт. Premium price - цена с надбавкой, цена с премией, премиальная цена. Цена товара или услуги, установленная на более высоком уровне, чем цены сходных товаров/услуг, предлагаемых конкурентами (например, цена, повышенная из-за наличия утовара/услуги уникальных особенностей либо вследствие интереса потребителей кданной торговой марке).

\section{ПРОГНОЗИРОВАНИЕ ВЫХОДА ГОДНЫХ}

Для прогнозирования и повышения выхода годных на ранних стадиях разработки технологии создаются тестовые макроструктуры на тестовых шаблонах. Выявление потенциальных производственных сбоев на ранней стадии разработки технологии позволяет проектным группам реализовать корректирующие действия и/или изменения процесса, которые сокращают время достижения желаемого показателя выхода годных. При стандартных методиках повышения выхода годных на новом уровне топологических норм технологического процесса в качестве отправной точки для идентификации структур для проектирования под эксперимент (DoE) используются конструкции предыдущего уровня топологий. Что же делать в случае применения новой технологии проектирования (например, многократного формирования рисунка), которой не было на предыдущих уровнях топологических норм технологического процесса?

Опыт человека-проектировщика в данном случае не подходит, так как нет знаний о подобных проблемах из предыдущих проектов. Кроме того, отсутствуют предварительные тестовые данные, на основе которых разработчики могут установить обратную связь для создания новых тестовых структур и определить средства оптимизации процесса или конструкции, способствующие более быстрому увеличению выхода годных.

Недавно появилась инновационная технология генерации топологической схемы (layout schema generation, LSG), которая позволяет проектным группам создавать дополнительные макроструктуры для их добавления в тестовые структуры, не полагаясь на вводимые данные предыдущих конструкций. Эти макроструктуры представляют собой случайное размещение единичных структур, на основе которых могут создаваться более крупные структуры. За счет 
использования нового LSG-процесса разработчики могут значительно сократить время достижения желаемого выхода годных для конструкций, при создании которых используются новые методики проектирования [1].

\section{ФАКТОРЫ, ВЛИЯЮЩИЕ НА НАДЕЖНОСТЬ И ВЫХОД ГОДНЫХ}

Выход годных пластин обычно уменьшается за счет трех категорий дефектов. К первой категории относятся случайные дефекты, возникающие из-за наличия загрязняющих частиц в различных технологических камерах. Проводящая частица может замкнуть две или больше соседних токопроводящих дорожек или создать канал утечки тока. Непроводящая частица или пустота способна разомкнуть проводящий канал или создать межсоединение с высоким сопротивлением.

Ко второй категории дефектов относятся систематические, возникающие из-за несовершенства архитектуры физического размещения элементов или влияния неоптимизированных способов оптических процессов и/или оборудования. Систематические дефекты, как правило, в наибольшей степени снижают выход годных, но большую их часть можно устранить методом оптимизации DTCO, когда конструкция и технологический процесс связываются более свободно, что позволяет быстрее получить желаемый результат. В третью категорию, которая не рассматривается в данной статье, входят параметрические дефекты (например, отсутствие воспроизводимости в процессе легирования), воздействующие на надежность приборов [1, 2].

\section{ГЕНЕРАЦИЯ ТОПОЛОГИЧЕСКОЙ СХЕМЫ}

Для демонстрации применимости LSG-процесса рассмотрим конструкции, использующие методики многократного формирования рисунка с SAMP. В настоящее время технология многократного формирования рисунка обычно применяется с ArF иммерсионной литографией (193-нм) - более предпочтительной по сравнению c EUV-литографией для новейших топологий порядка 20 нм и менее. На уровне 7/5-нм топологий процесс SAMP, по всей видимости, станет одной из наиболее эффективных методик многократного формирования рисунка с точки зрения достижения шага печатных линий на пластине, но выход годных при его использовании остается под вопросом. Разумеется, перед внедрением в серийное производство его необходимо опробовать на тестовых установках. Однако без наличия предшествующих конструкций SAMP проектирование соответствующих тестовых установок - сложная задача. В дополнениектому, что отсутствуют ретроспективные (накопленные) данные тестирования, однонаправленный характер SAMP-конструкций усложняет проектирование обычных «змеевидных» и «гребнистых» тестовых форм, которые содержат двунаправленные компоненты [1].

\section{ПРОЦЕСС МНОГОКРАТНОГО ФОРМИРОВАНИЯ РИСУНКА С САМОВЫРАВНИВАНИЕМ}

В процессе SAMP первый (первичный) шаблон известен как оправочный (mandrel mask). Жертвенные формы оправки печатаются с релаксированным шагом и после этого используются при проявке боковых стенок. Размеры боковых стенок составляют половину шага оправки. В зависимости от градации освещенности целевые формы могут существовать в промежутках между боковыми стенками. Целевые формы могут быть использованы повторно как жертвенные для формирования следующего поколения боковых стенок. Формы пластины, не имеющие соответствующих форм шаблона, называются неоправочными. Данный процесс можно повторять в целях достижения топологии SAMP с сокращенным шагом. Процесс SAMP (рис. 1) ограничивает конструкции до практической однонаправленности. Сгенерированные параллельные линии будут обрезаны позднее с использованием с шаблоном обрезки на месте желательного окончания линий - для формирования правильной подключаемости.

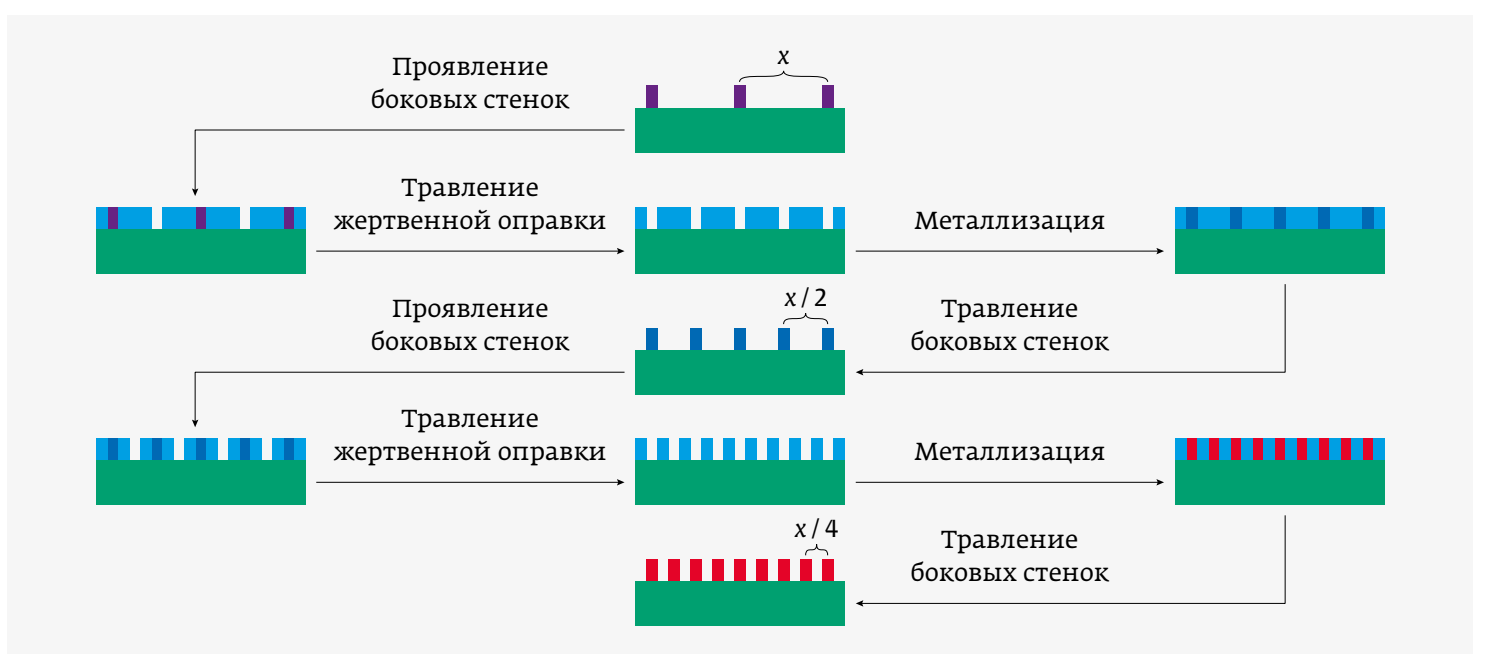

Рис. 1. Базовая схема процесса SAMP 


\section{ТЕСТОВЫЕ УСТАНОВКИ}

Тестовые установки обычно представляют собой подмножество шаблонов для проектирования, разработанных специально для того, чтобы активизировать потенциальные систематические отказы или литографические горячие точки в тестируемом слое. Тестовая установка также может содержать тестовые структуры, специально разработанные для обнаружения случайных дефектов. Другие структуры добавляются обычно на основе опыта, полученного при производстве кристаллов ИС предшествующих технологических поколений.

На новых топологических уровнях технологического процесса все тестовые структуры тестовой установки важны для процессов обучения и определения характеристик процесса. Обратная связь спроцессом тестирования используется для оптимизации методов проектирования. Например, когда "плохие" размеры топологических элементов обнаруживаются после завершения производства, они могут быть зафиксированы в виде структур, которым присвоены низкие оценки, и сохранены в библиотеках структур DFM". Затем с помощью DFM-анализа для поиска наихудших структур в данном топологическом чертеже проектировщики могут их изменить или устранить.

Ранняя оптимизация DTCO обеспечивает более быстрое достижение желаемого уровня выхода годных при освоении нового топологического уровня технологии. Отмечается, что даже на зрелых технологических уровнях тестовые структуры используются при производстве и обработке пластин для определения дополнительных возможностей совершенствования и оптимизации технологического процесса, что, в свою очередь, оказывает положительное влияние на выход годных.

Одно из препятствий при разработке средств тестирования состоит в том, что данный подход зависит, главным образом, от опыта и памяти человека, проектирующего тестовую систему. Хотя опытные специалисты знакомы с различными методиками проектирования на более зрелых технологических уровнях, но формы используемых структур (с которыми они встречаются) ограничены применяемыми методиками. Как правило, для разработки новых тестовых структур, особенно для нового процесса, требуется много времени. Решение LSG добавляет больше макроструктур (генерируемых случайным образом) в стандартную стратегию тестовых структур, что позволяет ускорить анализ влияния новых форм тестовых структур на выход годных.

DFM (design-for-manufacturing) - проектирование с обеспечением возможности непосредственного (прямого) внедрения (изделия) в производство (с точки зрения выхода годных, качества продукции и др.).

\section{СЛУЧАЙНАЯ ГЕНЕРАЦИЯ ТЕСТОВЫХ СТРУКТУР}

Основной компонент решения LSG - метод случайной генерации реалистичных, конструкционно-подобных топологических элементов без нарушения норм проектирования. B процессе LSG для обеспечения случайной генерации логическихзажимов (клипсов) для проверки топологии ИС за счет вставок базовых единичных структур в электрическую сеть конструкции используется метод Монте-Карло. Данные единичные структуры представляют собой простые прямоугольные и квадратные полигоны/элементы, а также единичные структуры для создания в конструкции промежутков между шагом полигонов/элементов. Размеры единичных структур зависят от значения шага конкретной технологии. При генерации топологий в качестве ограничений при вставке единичных структур применяются проектные нормы.

Для начала процесса SAMP нормы проектирования преобразуются в формат, читаемый автоматизированным LSG инструментальным средством наподобие Calibre LSG корпорации Mentor Graphics. Как только эти нормы СКонфигурированы, Calibre LSG-процесс может автоматически генерировать область реалистичных и свободных от нарушений проектных норм SAMP-структур произвольной ширины. Эта область ограничена только поуровневым планом заданного макроса SAMP тестовых структур. Тестовые структуры также могут быть сгенерированы с помощью шин электропитания для имитации топологий стандартных элементов.

\section{ПРОЕКТИРОВАНИЕ ПОД ЭКСПЕРИМЕНТ}

На этапе проектирования тестовой системы сгенерированные структуры SAMP добавляются к стандартному содержимому обычных тестовых структур. Случайные структуры SAMP электрически не имеют смысла, если они не соединены с другими слоями для постановки эксперимента. Методика DoE определяет правила подключения структур к тестовым контактным площадкам для обнаружения различных режимов отказов. К режимам отказов относятся короткие замыкания вследствие образования перемычек из-за недостатков процессов литографии или проводящих частиц, а также разрывы цепей вследствие нарушения целостности структур из-за недостатков литографии, непроводящих частиц, пустот или открытых межслойных переходов.

Для соединения случайных структур SAMP при проектировании под эксперимент (DoF) через слой трассировки к внешним контактным площадкам (для электрических измерений) может быть построена цепь межслойных переходов. Эти топологические клипсы для проверки ИС распределяются в соответствии с нормами разделения данной технологии на оправочные шаблоны и шаблоны обрезки. Разделенные клипсы могут тестироваться посредством моделирования или электрически на физическом уровне 


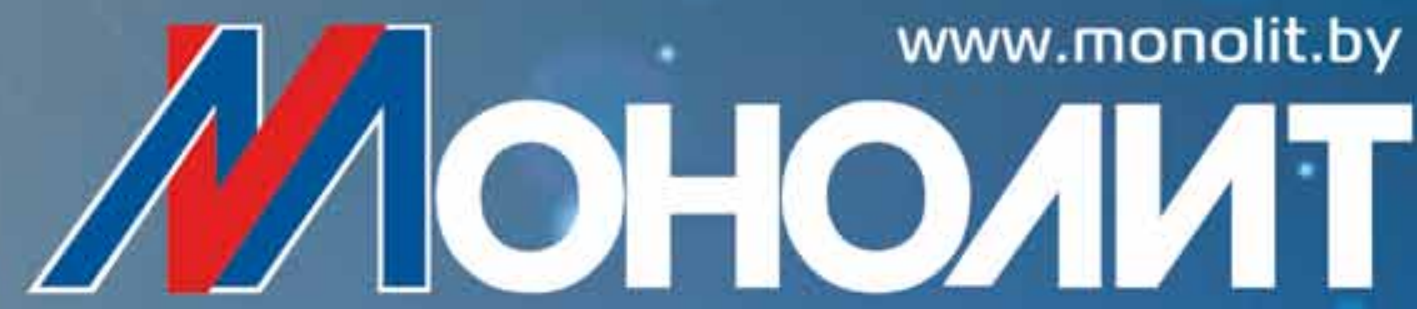

.

\section{МНОГОСАОЙНЫЕ КЕРАМИЧЕСКИЕ

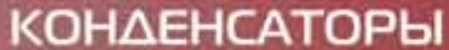

\section{ИМПОРТОВАМЕШАЮШАЯ ПРОАУКแИЯ}

мля высоконаАёжной аппаратуры

TЕРМОРЕЗИСТОРЫ сположительным температурным коэффишиентом сопротивления

21010I, РЕСПУБЛИКА БE ААРУСЬ г. Витебск, ул. М. Горького, 145

ОтАел маркетинга: Ten: + 375 (212) 36-44-52 факс: + 375 (212) 36-44-53 e-mail: monolmarket@mail.ru

Отьел продаж: тел:: +375 (212) 36-45-34 фakc: + 375 (212) 36-44-65 e-mail:monosbet@mail.ru

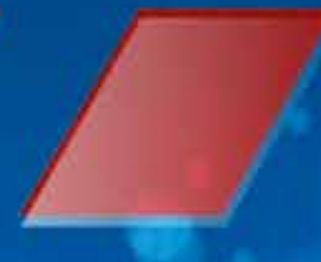


для выявления горячих точек. Горячие точки анализируются путем определения первопричины их возникновения. Впоследствии эта первопричина может учитываться при модификации топологии конструкции и/или оптимизации производственного процесса, а также моделей, которые позволят устранить данные горячие точки в производственных процессах поколений. Выявленные первопричины также можно использовать как обучающие структуры при разработке платформ DFM-правил. По мере увеличения размеров случайно генерируемых тестовых структур можно выявить больше горячих точек, что, в свою очередь, обеспечит ускорение выхода нового технологического уровня на желаемый уровень.

\section{ОБНАРУЖЕНИЕ СЛУЧАЙНЫХ ПРОВОДЯЩИХ ЧАСТИЦ}

В рамках эксперимента собрали данные о случайных дефектах, вызванных проводящими частицами. Все оправочные формы были присоединены через верхнее (или нижнее) межслойное соединение и металлические слои к тестовой контактной площадке, а неоправочные подключили таким же образом к другой тестовой контактной площадке. Верхний трассировочный слой формирует две встречно-гребенчатые формы, фрагмент топологического чертежа соединений показан на рис. 2. Межслойные переходы и верхняя металлическая трассировка были размещены с использованием специальных сценариев, без вмешательства человека-проектировщика. В идеале две тестовые контактные площадки должны быть разнесены так, чтобы оправочные формы не соприкасались с неоправочными. Если тестовые зонды соединяются, то это свидетельствует

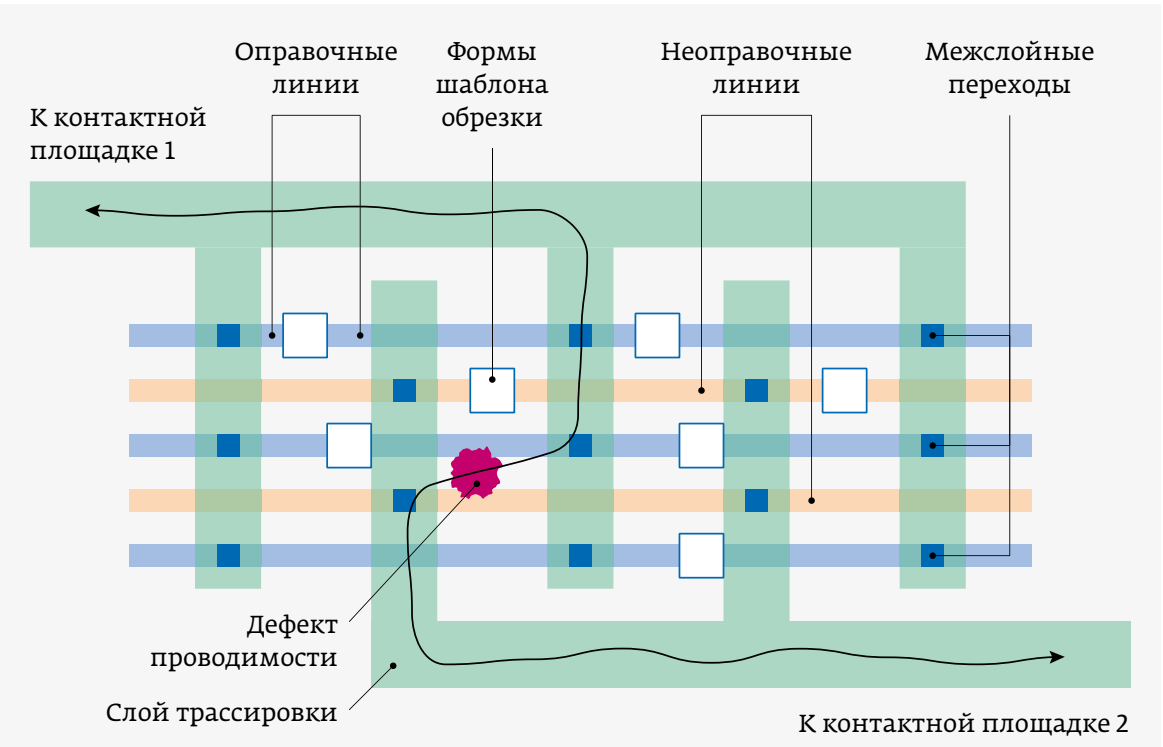

Рис. 2. Использование DoE для обнаружения дефектов, вызванных проводящими частицами о наличии дефекта проводящей частицы или литографической перемычки. Определение местоположения и анализ таких дефектов могут помочь в оценке и повышении уровня выхода годных.

\section{ОБНАРУЖЕНИЕ СИСТЕМАТИЧЕСКИХ ПРОБЛЕМ РАЗРЕШАЮЩЕЙ СПОСОБНОСТИ}

Один из примеров систематического дефекта литографии, найденного в конструкциях SAMP, состоит в том, что применение шаблонов и масок завершается некорректно. В результате две формы на одной и той же токопроводящей дорожке укорачиваются через незавершенный разрез. Тестирование в данном случае требует подключения других полигонов/элементов, находящихся на токопроводящей дорожке. Если окажется, что тестовые зонды соединены, а две контактные площадки (в идеальном случае) разъединены, это может означать незавершенность разреза. Анализ местонахождения данного дефекта и информации по партии из нескольких пластин поможет выявить его первопричину [1].

\section{БОЛЬШИЕ ДАННЫЕ КАК СРЕДСТВО УВЕЛИЧЕНИЯ ВЫХОДА ГОДНЫХ В ПРОЦЕССЕ ПРОИЗВОДСТВА}

Поставщики инструментальных средств и оборудования начинают рассматривать большие данные как средство увеличения выхода годных. Встраивание в процессе производства дополнительных датчиков и средств анализа помогает избежать возможных проблем. Насколько это повлияет на стоимость разработки сложных ИС по наиболее передовым технологическим нормам, а также 2,5D- и 3D-модулей, еще предстоит выяснить. Область разработок включает в себя создание датчиков, в бо́льшей степени соответствующих требованиям конкретных заказчиков/процессов, средств/методик машинного обучения и систем искусственного интеллекта (ИИ). Новые датчики быстро фиксируют данные, влияющие на текущие процессы, а также обеспечивают их долгосрочный сбор для выявления закономерностей. Кроме того, существует возможность своевременного отсева ненужных данных.

Движущий фактор развития предлагаемого подхода (оснащение производства дополнительными датчиками и средствами анализа) - оценка затрат на проект. По данным исследовательской корпорации International Business Strategies, стоимость разработки 5-нм специализированных ИC (ASIC) составляет 500 млн долл., а на 3-нм технологическом уровне 


\section{АКЦИОНЕРНОЕ ОБЩЕСТВО «ЗАВОД ПОЛУПРОВОДНИКОВЫХ ПРИБОРОВ»}

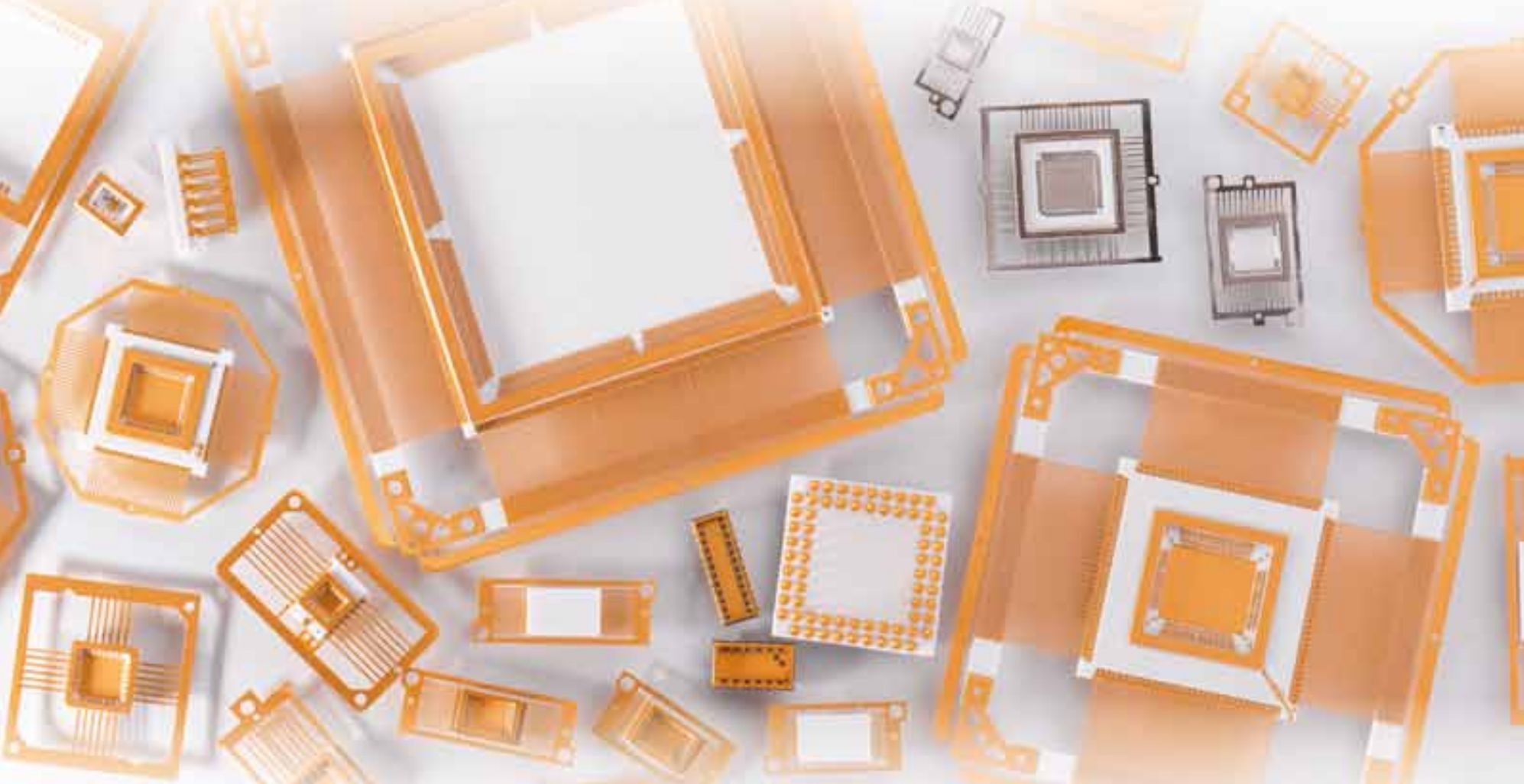

Предприятие располагает научно-технической и испытательной базой для проведения исследований, разработки и выпуска новой продукции

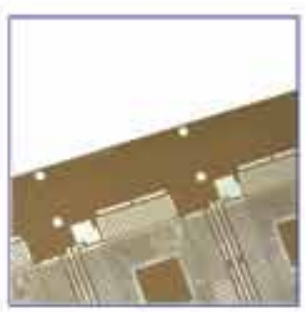

Выводные рамки

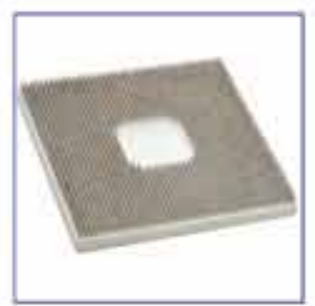

Mеталnокерамические корпуса

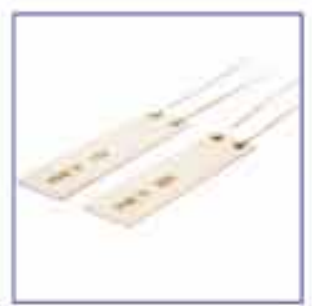

Harревательные элементы

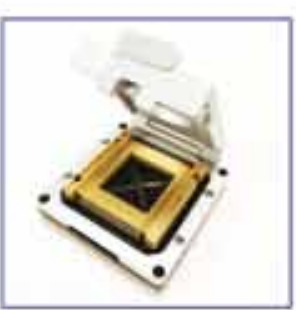

Контактные устройства

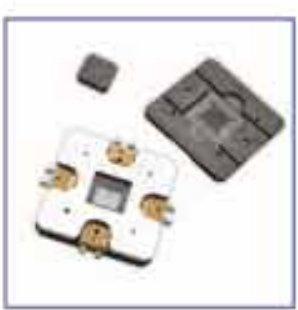

Графитовая оснастка

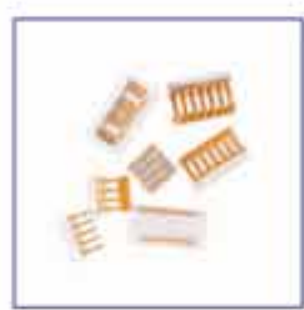

Оптозлектронные кopnyca

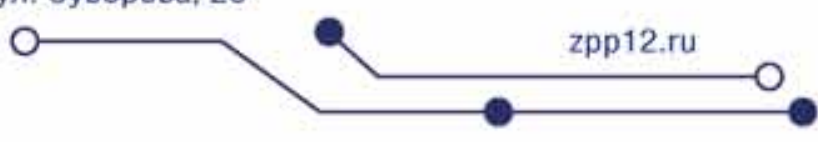


достигнет 1,5 млрд долл. На стадии разработки создаваемое изделие может пересматриваться в несколько этапов для улучшения функциональности и обеспечения соответствия первоначальному проекту ИС, формируемой на кристалле. С одной стороны, существует ряд возможных способов снижения стоимости проекта и сокращения времени от начала разработки до вывода изделия на рынок, а с другой - растет спрос разработчиков подобных ИС на реализацию этих возможностей $[3,4]$.

По мере уменьшения минимальных размеров топологических элементов схемы становятся более чувствительными к дефектам. Соответственно, оснащение производственных линий средствами их обнаружения не только необходимо, но и полезно. Так, благодаря наличию подобных средств в I квартале 2019 года корпорации TSMC удалось своевременно снять с обработки партию пластин из-за проблем с сопротивлением. Ранее подобная партия могла бы пройти весь цикл обработки и выйти на рынок.

Проблемы, связанные с различными типами дефектов, усугубляются по мере увеличения плотности расположения элементов и снижения их топологических размеров. То, что не было проблемой на уровне 28-нм технологий, например, несовершенно сформированный топологический элемент, на уровне 7-нм технологий может стать причиной явного или скрытого дефекта. На передовых технологических уровнях управление технологическим процессом должно быть связано с проектным допуском, будь то FinFET-конструкция или конструкция FinFET+3D. В настоящее время функции обнаружения и классификации отказов (fault detection and classification, FDC) и датчики отделены от конструкции. Однако по мере продвижения при рассмотрении топологии и допусков их необходимо увязывать с возможностями процесса. Начальные этапы реализации концепции Industry 4.0" и Ии обеспечили некоторые успехи, но теперь проектировщики больше внимания уделяют наличию датчиков и достоверных данных, которые можно увязать с конкретным процессом. Это имеет большое значение для сокращения времени вывода нового (модернизированного) продукта на рынок (time-to-market).

Industry 4.0 (The Fourth Industrial Revolution) - 4-я промышленная революция, ожидаемое массовое внедрение киберфизических систем в производство и обслуживание человеческих потребностей, включая быт, труд и досуг. Также это производственная сторона, эквивалентная ориентированному на потребителей Интернету вещей. Один из существенных аспектов Industry 4.0 - идея сервис-ориентированного проектирования (от пользователей, предпочитающих заводские настройки для производства собственных продуктов, до компаний, поставляющих потребителям индивидуальные продукты).
Важно не только понимать, какие данные полезны, но и уметь использовать данные, передаваемые датчиками. Этих устройств может быть очень много - встроенные и невстроенные, метрологические и датчики инструментальных средств и т. д

Кроме того, есть функциональные данные (в какие камеры и какие пластины направляют роботизированные манипуляторы и т.п.). Набор данных определяют пределы действия инструментального средства или окружающей вычислительной среды.

Для различных этапов процесса характерны различные допуски. В прошлом на каждом этапе мог быть свой допуск, при этом конечное изделие работало. С каждым этапом формирования рисунка накапливается бюджет ошибок, поэтому требуется более жесткий контроль. Кроме того, необходимы более частые выборки данных и получение бо́льшего объема информации. Ранее для определения характеристик слоя в процессе его обработки можно было использовать от 10 до 30 точек данных. Сегодня нужны бо́льший объем выборок, а также прямая и обратная передача данных. Это приводит к большому объему данных для определения характеристик изменчивости по всей пластине или кристаллу.

Генерация больших объемов данных требует использования локальных серверов или облачных вычислений. Для бо́льшей части производственных операций это было бы промежуточным этапом между датчиками и облачным хранилищем. Но кремниевые заводы отказываются хранить любые данные в общедоступных облаках (требования защиты данных и интеллектуальной собственности). В результате заводам по обработке пластин самим приходится анализировать, какие данные обрабатывать немедленно, а какие позже.

Размеры формируемых на пластине структур измеряются на уровне ангстрема, поэтому допуски на изменчивость параметров намного более жесткие. С точки зрения контроля изменчивость проявляется по-разному: от партии к партии пластин, от пластины к пластине, собственно на пластине, на уровне кристалла, на уровнях неравномерности краев линии (резиста)/неравномерности ширины линии / локальной воспроизводимости критических размеров - LER / LWR / LCDU. Hекоторые используемые точки данных рассматриваются как возможность повышения степени контроля процесса. При этом одна из проблем для производителей связана с пониманием того, как использовать полученные данные. Важный фактор - подключение как можно большего числа источников метрологической контрольной информации. Если удается стабилизировать процесс на этапе НИОКР, то можно уменьшить количество параметров / выборок, необходимых для обеспечения надежности $[3,5]$. 


\section{ОСЦИЛЛОГРАФЫ АКИП}

ОСЦИЛЛОГРАФЫ

СТРОБОСКОПИЧЕСКИЕ

\section{АКИП-4132 - 9 моделей}

- Полоса пропускания:

15 ГГц, 20 ГГц, 25 ГГц

- 2 или 4 канала

- Частота стробирования 1 МГц

- Модели АКИП-4132/5, АКИП-4132/6

имеют встроенный генератор

импульсов с фронтом 60 пс

\section{АКИП-4112 - 7 моделей}

- Полоса пропускания: 12 ГГц, 20 ГГц

- 2 или 4 канала

- Частота стробирования 1 МГц

\section{УНИКАЛЬНАЯ НОВИНКА \\ АКИП-4133}

- Осциллограф реального времени до 500 МГц

- Осциллограф стробоскопический до 16 ГГц

- АЦП 12 бит

- Внутренняя синхронизация до 2,5 ГТц, с делителем частоты до 6 ГГц

- Автоматизированный тест сигнала по маске (>100 предустановленных шаблонов по стандартам SONET/ $\mathrm{SDH}$, Fiber Channel, Ethernet, Infiniband, XAUI, ITU G.703, ANSI T1/T102, RapidIO, PCl Express, Serial ATA)

- Интерфейсы: USB, LAN

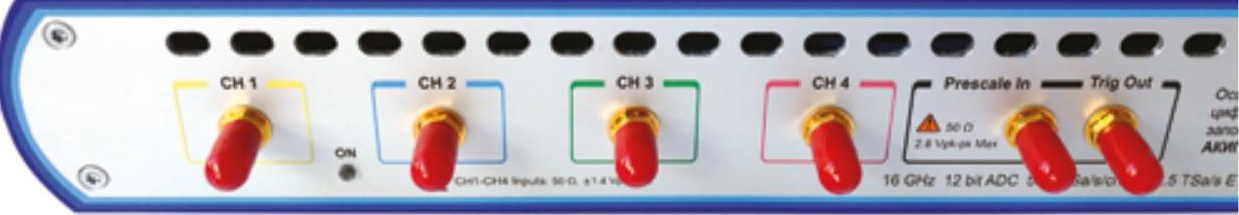

\section{ОСЦИЛЛОГРАФЫ РЕАЛЬНОГО ВРЕМЕНИ до 1 ГГц}

АКИП-4134 1 ГГц, 2/4 канала, дискретизация 5 Гвыб/с АКИП-4126X 300 МГц, 2/4 канала, дискретизация 2 Гвыб/с АКИП-4126Е 350 МГц, 2 канала, дискретизация 2 Гвыб/с АКИП-4131 200 МГц, 2/4 канала, дискретизация 1 Гвыб/с АКИП-4122 300 МГц, 2 канала, дискретизация 3,2 Гвыб/с АКИП-4115 200 МГц, 2 канала, дискретизация 1 Гвыб/с

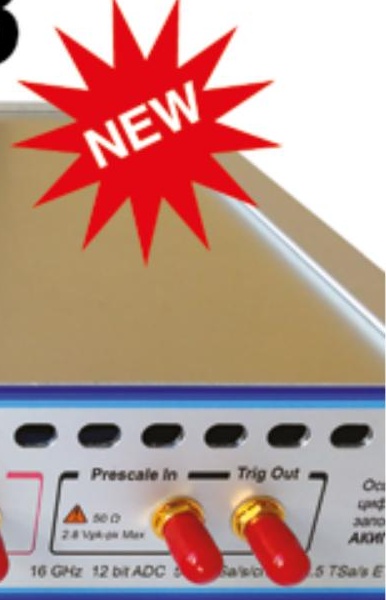




\section{ИЗМЕНЧИВОСТЬ ПАРАМЕТРОВ ПРОЦЕССОВ / ПРИБОРОВ}

В полупроводниковой промышленности затрачиваются большие средства на то, чтобы свести к минимуму изменчивость параметров процессов/приборов. Конструкции ИС становятся все сложнее, число переменных растет, а потенциал изменчивости каждой из них порождает возможность взрывообразного роста различных дефектов.

Для решения проблемы изменчивости предлагается множество вариантов компенсаций в различных схемах контроля / управления процессами. Появление FinFET вызвало трудности - трехмерная природа этой структуры «бросила вызов" традиционным подходам, и промышленность ищет надежные решения для поточно-массового производства.

При топологических размерах элементов порядка 5 нм все еще сложнее. При выходе на структуры следующего поколения с топологиями 3 нм и менее (круговые затворы, gate-all-around) сложность увеличивается еще на порядок. На первый взгляд, данный переход выглядит как модификация FinFET, но требования повышаются, а по сложности эта архитектура затвора значительно превосходит FinFET. Более сложные структуры появляются при каждом технологическом переходе на новый уровень с меньшими топологиями, и они становятся источниками изменчивости параметров процессов/приборов.

Но наряду с этим существует множество других источников изменчивости параметров - от видов примесей в используемых материалах до способов применения этих материалов в технологическом процессе.

Так, например, на каждом новом технологическом уровне толщина пленок должна контролироваться с гораздо большей точностью, чем на предшествующем, поскольку допуски становятся все более жесткими. В то же время выявить отклонения параметров процесса с использованием имеющихся инструментальных средств контроля все труднее.

Один из актуальных вопросов - охват области сбора данных. Зачастую неизвестно, в чем состоят и где возникают проблемы, поэтому требуются дополнительные выборки и бо́льший объем данных. Если речь идет о разрешении порядка 1 нм на уровне пластины, то объем данных равен $10^{12}$ пикселей. По мере поступления такие огромные объемы данных нуждаются в фильтрации: одни надо накапливать, другие - анализировать сразу, сравнивая с другими данными. Главное - знать, где искать проблемы, потому что не все можно проверить и измерить $[3,4]$.

\section{НОВЫЕ ПРОБЛЕМЫ И ПУТИ ИХ РЕШЕНИЯ}

Противодействие изменчивости параметров процессов/продуктов и, соответственно, дефектности должно происходить в реальном масштабе времени. Задержки с освоением новой продукции на заводе по обработке пластин не только увеличивают издержки производства, но и могут повлиять на возможность продаж кристаллов ИС. Кроме того, нарушаются графики выпуска на рынок новых конструкций конечных электронных систем, оснащаемых ИС, сужаются их рыночные возможности. С другой стороны, прохождение сквозь сито контроля дефектов может вызвать другие проблемы, особенно когда речь идет о ИС, разработанных на базе передовых технологий для автомобильной, медицинской и промышленной электроники.

Отраслевые специалисты выделяют несколько методов снижения уровня дефектности. Один из них заключается в тщательном контроле процесса с помощью программ его непрерывного совершенствования для уменьшения случайных дефектов. В этом случае используются базовые методы повышения выхода годных, такие как мониторинг инструментальных средств в процессе обработки. Другой метод предусматривает осуществление выборок данных на уровне, достаточном для обеспечения отслеживаемости процесса.

В последнее время все больший интерес вызывает метод, основанный на получении информации о дефектах от встроенных в производственную линию средств метрологии. Подобная информация используется не только для контроля технологического процесса, но и для выявления потенциальных проблем с надежностью, пока кристаллы находятся на заводе по обработке пластин, где исправление дефектов обходится дешевле. Производители автомобильных полупроводниковых приборов давно полагаются на метод скрининга (screening), в рамках которого инструмент с высокой пропускной способностью проверяет все кристаллы ИС на всех пластинах в конце производственного процесса (при этом количество слоев в завершенном кристалле ИС может достигать 10-12). Кристалл, отвечающий определенным критериям отказа (размер/тип/местоположение дефекта), выбраковывается или специально помечается для дальнейшего использования в системах с меньшими нагрузками и требованиями к надежности. Метод эффективен для выявления крупных дефектов, но недостаточен для обнаружения небольших скрытых дефектов $[3,5,6]$.

Решением может стать новая встроенная в технологический процесс методика усредненного (обычного) тестирования приборов в процессе обработки/ формирования (inline parts average testing, І-РАТ). Методика, известная как усредненный параметрический контроль приборов (parametric parts average testing, РРАТ), используется в автомобильной промышленности более 20 лет. Первоначальный метод, основанный на электронном тестировании, позволяет идентифицировать любой кристалл, результаты тестирования которого вне нормального распределения концентрации дефектов, даже если они находятся в пределах эксплуатационных спецификаций. Методика І-РАТ позволяет использовать эту концепцию непосредственно в технологическом 


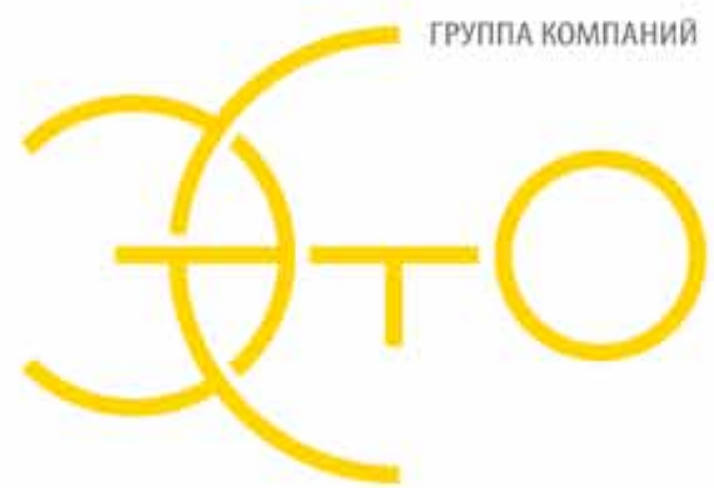

Акционерное общество «Научно-промзводственное предприятие «электронное

cneциальное технологическое оборудованиеу

ЭЛЕКТРОННОЕ СПЕЦИАЛЬНОЕ ТЕХНОЛОГИЧЕСКОЕ ОБОРУДОВАНИЕ

АО НППЭСТО (Группа компаний ЭСТО) - объединение ведущих российских предприятий, специализирующихся на разработках, производстве, модернизации, продаже и сервисном обслуживании специального технологического оборудования.

Направления деятельности группы «ЭСТО».

Разработка и производство технологического оборудования (лазерное, вакуумное, сборочное, нестандартное) и внедрение технологий

Организация поставок как отдельных единиц зарубежного технологического оборудования, так и комплексных законченных технологий «под ключ»)

Комплексная и частичная модернизация российского и зарубежного технологического оборудования любой сложности

Сервисное обслуживание российского и зарубежного технологического оборудования

Проектирование и строительство производств микроэлектроники

Обучение специалистов заказчика

Технологический аудит производства

124460, Москва, Зеленоград. просп. Георгиевский, д. S, стр. 1 тел. (499) 729-77-51, (499) 479-12-39 info@inppesto.ru www.nppestoru

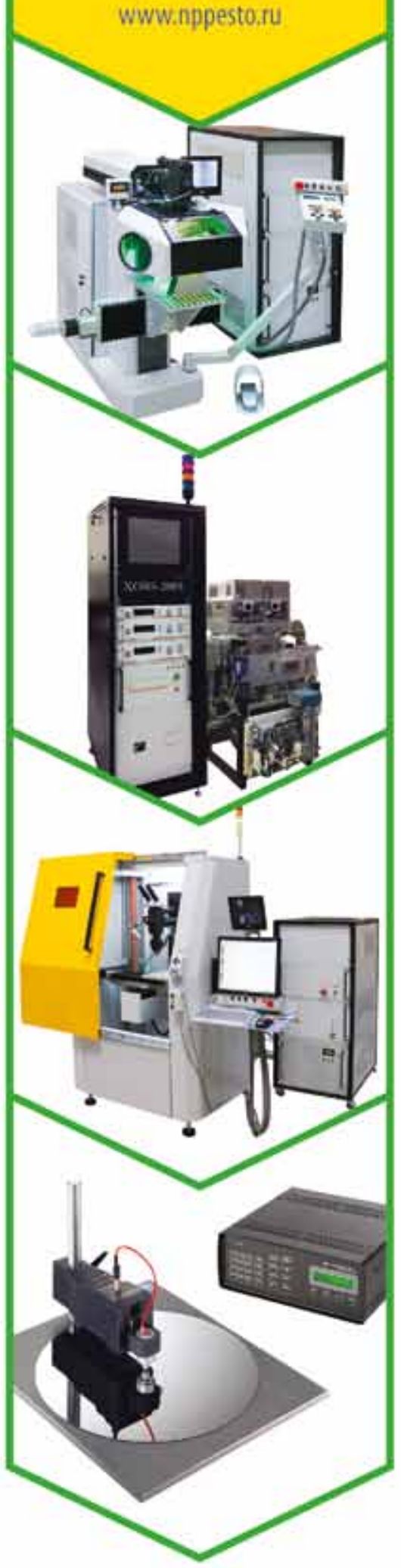




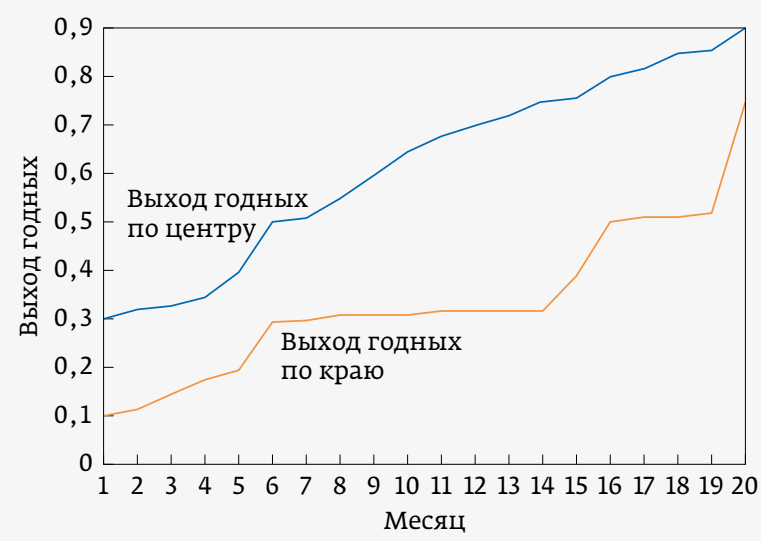

процессе для поиска кристаллов с критичными концентрациями дефектов в рамках многократных этапов контроля на протяжении производственного процесса.

С точки зрения статистики скрытые дефекты, которых необходимо избегать, легче найти по выбросам / провалам на графике, построенном по информационным точкам. Для проверки полученной информации ее можно совместить с данными, полученными электрическими методами, что уточнит критерии для принятия решения "подходит / не подходит» по кристаллу [4]

Отмечается, что выход годных может зависеть от того, в каком месте пластины размещаются кристаллы, а также от диаметра обрабатываемой пластины (рис. 3).

По мере того как все больше фирм-интеграторов конечных электронных систем участвует в разработке ИС, реализованных по новейшим технологиям с минимальными размерами топологических элементов, все больше внимания уделяется тому, чтобы выпускаемое изделие не отличалось от проектируемого. Однако подобный подход выходит за рамки только процесса проектирования, поскольку охватывает всю цепочку поставок для ИС, формируемых по передовым технологиям

Еще одна проблема - дефектность шаблонов. Если нет бездефектных заготовок шаблона с нанесенным слоем резиста, не будет и бездефектного шаблона. При этом некоторые дефекты будут незначительны, и речь может идти не о выбраковке кристаллов, а о переопределении сферы их конечного применения. Отдельные дефекты можно даже исправить. Производители расходуют значительные средства для того, чтобы создать надежные методы, позволяющие отличить некритичные дефекты от катастрофических, так как подобные подходы - один из способов увеличения выхода годных.

В процессе проектирования основное внимание уделяется возможности воспроизводимости рисунка. Для исправления незначительных дефектов используется метод коррекции эффекта оптической близости (optical proximity correction, OPC) $[3,4]$.

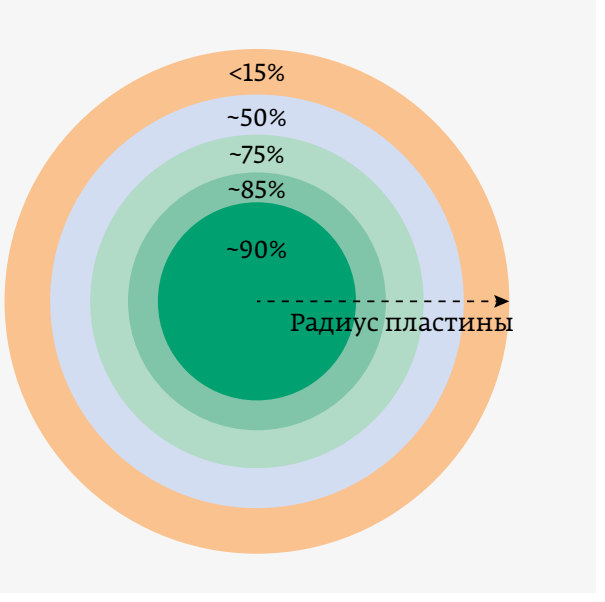

Рис. 3.

Сопоставление выхода годных по центру и по краю пластины во время освоения нового прибора как функция времени

По мере дальнейшего масштабирования приборов, увеличения плотности расположения их элементов становится все более необходимым применение методик больших данных, которые позволяют максимально оперативно выявить возникновение дефектов на ранних этапах технологического процесса.

\section{ЛИТЕРАТУРА}

1. Khakifirooz M., Chien Ch.F., Chen Y.-J. Bayesian Inference for Mining Semiconductor Manufacturing Big Data for Yield Enhancement and Smart Production to Empower Industry 4.0. Applied Soft Computing. November 2017. https://www. researchgate.net/publication/321304885_Bayesian_Inference_ for_Mining_Semiconductor_Manufacturing_Big_Data_for_Yield_ Enhancement_and_Smart_Production_to_Empower_Industry_40.

2. Sperling E. Using Sensor Data To Improve Yield And Uptime // Semiconductor Engineering. February 21st. 2019.https:// semiengineering.com/using-sensor-data-to-improve-chipmanufacturing-and-yield/.

3. Sperling E. Controlling IC Manufacturing Processes For Yield // Semiconductor Engineering. April 24th. 2019. https:// semiengineering.com/controlling-ic-manufacturing-processes-toboost-yield/.

4. Elmanhawy W., Kwan J. Layout schema generation: Improving yield ramp during technology development // Solid State Technology. Wafer News. November 20. 2018. https://electroiq.com/2018/11/ layout-schema-generation-improving-yield-ramp-during-technologydevelopment/.

5. Nakata K., Orihara R., Mizuoka Y. A Comprehensive Big-DataBased Monitoring System for Yield Enhancement in Semiconductor Manufacturing // IEEE Transactions on Semiconductor Manufacturing. V. 30, Is. 4. Nov. 2017. https://ieeexplore.ieee.org/ document/8039264.

6. Chien Ch.-F., Liu Ch.W., Chuang Sh.-Ch. Analysing semiconductor manufacturing big data for root cause detection of excursion for yield enhancement //International Journal of Production Research. V. 55. 2017. Is. 17. https://www.tandfonline.com/doi/abs/10.1080/ 00207543.2015 .1109153$. 


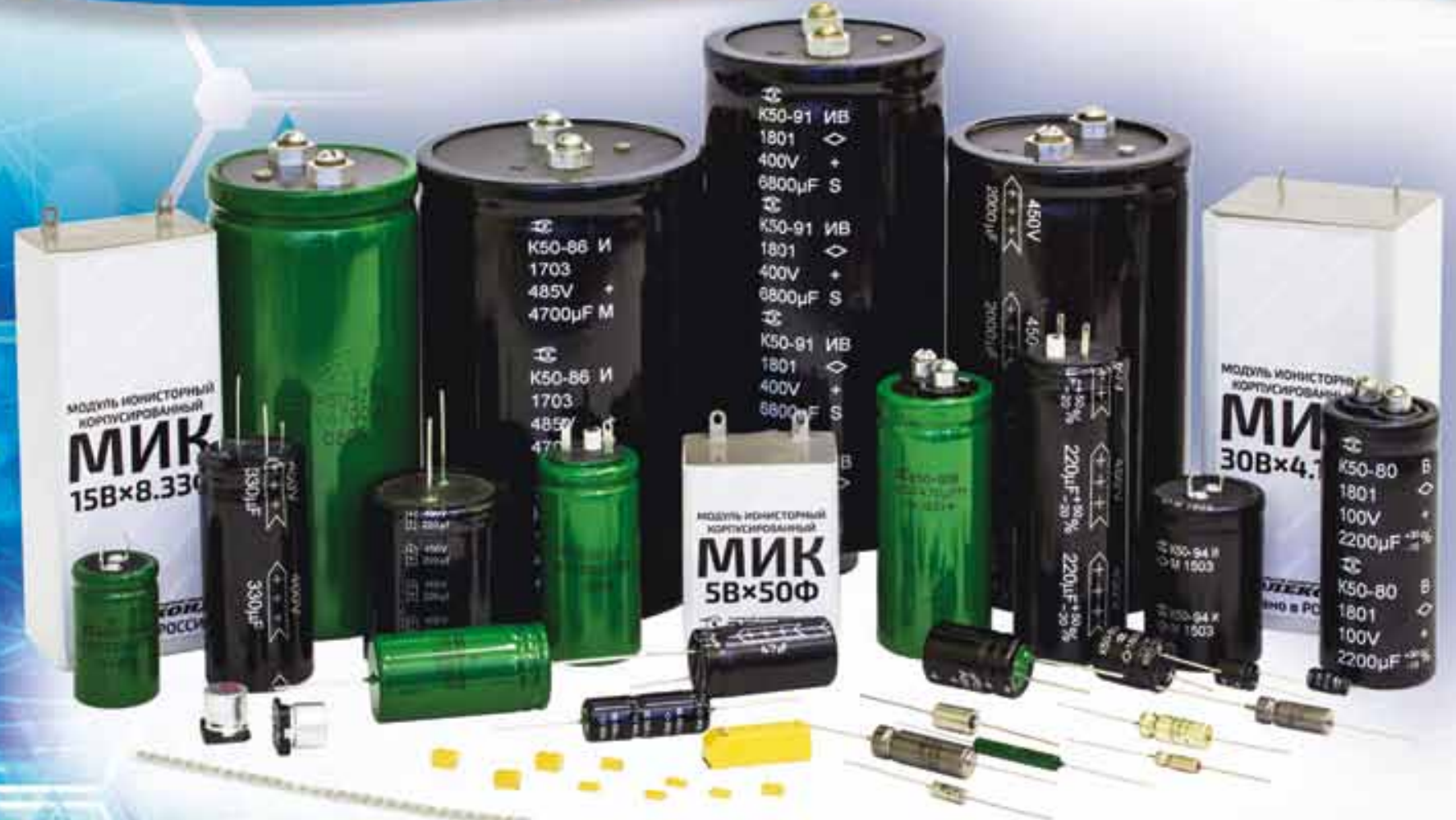

\section{Разработка и производство конденсаторов}

оксидно-электролитические алюминиевые конденсаторы К50-15, К50-17, К50-27, К50-37, К50-68, К50-74, К50-76, К50-77, К50-80, К50-81, К50-83, К50-84, К50-85, К50-86, К50-87, К50-88, К50-89, К50-90, К50-91, К50-92, К50-93, К50-94, К50-95(чип), К50-96, К50-98

объемно-пористые танталовые конденсаторы К52-1, К52-1М, К52-1БМ, К52-1Б, К52-9, К52-11, К52-17, К52-18, К52-19, К52-20, К52-21, К52-24, К52-26(чип), К52-27(чип)

оксидно-полупроводниковые танталовые конденсаторы

К53-1А, К53-7, К53-65(чип), К53-66, К53-68(чип), К53-71(чип), К53-72(чип), К53-74(чип), К53-77(чип), К53-78(чип)

суперконденсаторы (ионисторы) K58-26

накопители электрической энергии на основе модульной сборки суперконденсаторов

Система менеджмента качества сертифицирована на соответствие требованиям ISO 9001

Россия, 427968, Удмуртская Республика, г. Сарапул, ул. Калинина, 3 Тел.: (34147) 2-99-53, 2-99-89, 2-99-77, факс: (34147) 4-32-48, 4-27-53 e-mail: elecond-market@elcudm.ru, http://www.elecond.ru 